PROCEEDINGS OF THE

AMERICAN MATHEMATICAL SOCIETY

Volume 124, Number 12, December 1996, Pages 3721-3727

S 0002-9939(96)03879-8

\title{
THE LUSIN-PRIVALOV THEOREM FOR SUBHARMONIC FUNCTIONS
}

\author{
STEPHEN J. GARDINER
}

(Communicated by Albert Baernstein II)

\begin{abstract}
This paper establishes a generalization of the Lusin-Privalov radial uniqueness theorem which applies to subharmonic functions in all dimensions. In particular, it answers a question of Rippon by showing that no subharmonic function on the upper half-space can have normal limit $-\infty$ at every boundary point.
\end{abstract}

\section{INTRODUCTION}

Let $u$ be a subharmonic function on the upper half-plane $D$ and let

$$
A=\{x \in \mathbb{R}: u(x, y) \rightarrow-\infty \text { as } y \rightarrow 0+\} .
$$

Then $A \neq \mathbb{R}$. Indeed, the Lusin-Privalov radial uniqueness theorem for analytic functions [12] has a generalization for subharmonic functions on $D$ (see [1], [3], [13]) which asserts that, if $A$ is metrically dense in an open interval $I$ (i.e. $A$ has positive linear measure in each subinterval of $I$ ), then $A \cap I$ is of first category.

Rippon [13, Theorem 6] showed that this result breaks down in higher dimensions by constructing a subharmonic function $u$ on $\mathbb{R}^{2} \times(0,+\infty)$ such that

$$
u\left(x_{1}, x_{2}, x_{3}\right) \rightarrow-\infty \quad\left(x_{3} \rightarrow 0+;\left(x_{1}, x_{2}\right) \in \mathbb{R}^{2} \backslash E^{\prime}\right),
$$

where $E^{\prime}$ is a first category subset of $\mathbb{R}^{2}$ with zero area measure. A key observation here is that a line segment is polar in higher dimensions but not in the plane. One way around this problem is to replace normal limits by limits along translates of a somewhat "thicker" set, as in [13]. However, this leaves open the question, posed in [13, p. 479], of whether a subharmonic function on the upper half-space can have normal limit $-\infty$ at every boundary point. In this paper we give a negative answer to this question by establishing a suitable higher dimensional generalization of the Lusin-Privalov theorem.

The fine topology on $\mathbb{R}^{n}$ is the coarsest topology which makes every subharmonic function continuous. We refer to Doob [8, 1.XI] for its basic properties. Let $U$ be a non-empty fine open set. A set $A$ is said to be metrically fine dense in $U$ if, for every non-empty fine open subset $V$ of $U$, the set $A \cap V$ has positive outer $\lambda_{n^{-}}$ measure, where $\lambda_{n}$ denotes Lebesgue measure on $\mathbb{R}^{n}$. Also, $A$ is said to be of first fine category if it can be expressed as a countable union of sets $E_{k}$ such that the fine closure of each $E_{k}$ has empty fine interior. These definitions are given substance by

Received by the editors May 10, 1995.

1991 Mathematics Subject Classification. Primary 31B25.

(C)1996 American Mathematical Society 
the observations that every non-empty fine open set has positive $\lambda_{n}$-measure, and that the fine topology has the Baire property: see $\S 3.2$.

Points of $\mathbb{R}^{n}(n \geq 2)$ will be denoted by $X$, or by $\left(X^{\prime}, x\right)$ where $X^{\prime} \in \mathbb{R}^{n-1}$, and the upper half-space $\mathbb{R}^{n-1} \times(0,+\infty)$ will be denoted by $D$. Our generalization of the Lusin-Privalov theorem is as follows.

Theorem 1. Let $u$ be a subharmonic function on $D$ and let $U^{\prime}$ be a non-empty fine open subset of $\mathbb{R}^{n-1}$. If the set

$$
\left\{X^{\prime} \in \mathbb{R}^{n-1}: \liminf _{x \rightarrow 0+} u\left(X^{\prime}, x\right)=-\infty\right\}
$$

is metrically fine dense in $U^{\prime}$, then the set

$$
\left\{X^{\prime} \in U^{\prime}: \limsup _{x \rightarrow 0+} u\left(X^{\prime}, x\right)<+\infty\right\}
$$

is of first fine category (in $\left.\mathbb{R}^{n-1}\right)$.

Corollary 1. If $U^{\prime}$ is a non-empty fine open subset of $\mathbb{R}^{n-1}$, then there is no subharmonic function $u$ on $D$ such that

$$
u\left(X^{\prime}, x\right) \rightarrow-\infty \quad\left(x \rightarrow 0+; X^{\prime} \in U^{\prime}\right) .
$$

The fine topology on $\mathbb{R}^{n}$ is strictly finer than the Euclidean one when $n \geq 2$. This is not true when $n=1$, since the subharmonic functions on $\mathbb{R}$ are precisely the convex functions and hence are already continuous. Thus, when $n=2$, Theorem 1 is only a slight refinement of the result cited at the beginning of the paper. However, when $n \geq 3$, Theorem 1 is new and, in view of Corollary 1 , gives a negative answer to the question of Rippon noted above.

A variant of the Lusin-Privalov theorem, due to Barth and Schneider [2] has the following generalization to higher dimensions.

Theorem 2. Let $f:(0,1] \rightarrow \mathbb{R}$ be such that $f(x) \rightarrow-\infty$ as $x \rightarrow 0+$, and let $u$ be a subharmonic function on $D$. Then the set

$$
E^{\prime}=\left\{X^{\prime} \in \mathbb{R}^{n-1}: \limsup _{x \rightarrow 0+}\left\{u\left(X^{\prime}, x\right)-f(x)\right\}<+\infty\right\}
$$

is of first fine category.

Let $\rho$ denote the metric on $[-\infty,+\infty]$ given by $\rho(x, y)=\left|\tan ^{-1} x-\tan ^{-1} y\right|$. The closure of a subset $A$ of $[-\infty,+\infty]$ with respect to $\rho$ will be denoted by $\bar{A}^{\rho}$. Given a point $X^{\prime}$ in $\mathbb{R}^{n-1}$ and a function $g: D \rightarrow[-\infty,+\infty]$ we define the normal and fine cluster sets of $g$ at $\left(X^{\prime}, 0\right)$ by

$$
C_{N}\left(g, X^{\prime}\right)=\bigcap_{t>0}{\overline{\left\{g\left(X^{\prime}, x\right): 0<x<t\right\}}}^{\rho}
$$

and

$$
C_{F}\left(g, X^{\prime}\right)=\bigcap_{V} \overline{\{g(Y): Y \in V \cap D\}}{ }^{\rho}
$$

respectively, where the latter intersection is over all fine neighbourhoods $V$ of $\left(X^{\prime}, 0\right)$ in $\mathbb{R}^{n}$. The minimal fine cluster set $g$ at $\left(X^{\prime}, 0\right)$, denoted by $C_{M}\left(g, X^{\prime}\right)$, is defined analogously with respect to the minimal fine topology for $D$ (see $[8,1$.XII] for an account of this topology). 
Theorem 3. If $g: D \rightarrow[-\infty,+\infty]$ is fine continuous, then there is a first fine category subset $E^{\prime}$ of $\mathbb{R}^{n-1}$ such that

$$
C_{F}\left(g, X^{\prime}\right) \subseteq C_{N}\left(g, X^{\prime}\right) \quad\left(X^{\prime} \in \mathbb{R}^{n-1} \backslash E^{\prime}\right) .
$$

Theorem 3 is similar in spirit to a classical result of Collingwood [4, p. 76] concerning boundary cluster sets of continuous functions on the unit disc. When $n=2$ a stronger result than Theorem 3 is true; namely, $C_{N}\left(g, X^{\prime}\right)$ is equal to the full cluster set of $g$ at $\left(X^{\prime}, 0\right)$ for all but a first category set of points $X^{\prime}$ in $\mathbb{R}$; see $[13$, Theorem 1].

Doob [7, Theorem 4.1] has shown that, for any function $g: D \rightarrow[-\infty,+\infty]$, the inclusion $C_{N}\left(g, X^{\prime}\right) \subseteq C_{M}\left(g, X^{\prime}\right)$ holds for $\lambda_{n-1}$-almost every $X^{\prime}$ in $\mathbb{R}^{n-1}$. In the opposite direction we can now give the following.

Corollary 2. If $g: D \rightarrow[-\infty,+\infty]$ is fine continuous, then there is a first fine category subset $E^{\prime}$ of $\mathbb{R}^{n-1}$ such that

$$
C_{M}\left(g, X^{\prime}\right) \subseteq C_{N}\left(g, X^{\prime}\right) \quad\left(X^{\prime} \in \mathbb{R}^{n-1} \backslash E^{\prime}\right) .
$$

The arguments used to prove Theorem 3 and Corollary 2 form part of the proofs of Theorems 1 and 2, so we begin by proving Theorem 3 in $\S 2$. Theorems 1 and 2 are then proved in $\S 3$ and $\S 4$ respectively. Finally, we given an example relating to these results in $\S 5$. Since our results are new only when $n \geq 3$, we will restrict our attention to this case in what follows.

\section{Proof of Theorem 3 and Corollary 2}

2.1. A set $A$ in $\mathbb{R}^{n}$ is said to be thin at a point $X$ if $X$ is not a fine limit point of $A$. We begin with two preparatory lemmas, the first of which is known (see [5, Lemme 2]).

Lemma 1. Let $A^{\prime} \subseteq \mathbb{R}^{n-1}$. Then $A^{\prime} \times \mathbb{R}$ is polar in $\mathbb{R}^{n}$ if and only if $A^{\prime}$ is polar in $\mathbb{R}^{n-1}$.

Lemma 2. Let $A^{\prime} \subseteq \mathbb{R}^{n-1}$ and $\left(Y^{\prime}, y\right) \in \mathbb{R}^{n}$. Then $A^{\prime} \times \mathbb{R}$ is thin at $\left(Y^{\prime}, y\right)$ if and only if $A^{\prime}$ is thin at $Y^{\prime}$.

To prove Lemma 2, suppose that $A^{\prime}$ is thin at $Y^{\prime}$. If $Y^{\prime}$ is not a limit point of $A^{\prime}$, then there is a (Euclidean) neighbourhood $U^{\prime}$ of $Y^{\prime}$ such that $\left(U^{\prime} \cap A^{\prime}\right) \times \mathbb{R}$ is contained in the polar set $\left\{Y^{\prime}\right\} \times \mathbb{R}$, and so $A^{\prime} \times \mathbb{R}$ is thin at $\left(Y^{\prime}, y\right)$. If $Y^{\prime}$ is a limit point of $A^{\prime}$, then there is a superharmonic function $u^{\prime}$ on $\mathbb{R}^{n-1}$ such that

$$
u^{\prime}\left(Y^{\prime}\right)<\liminf _{\substack{X^{\prime} \rightarrow Y^{\prime} \\ X^{\prime} \in A^{\prime}}} u^{\prime}\left(X^{\prime}\right)
$$

If we define $u\left(X^{\prime}, x\right)=u^{\prime}\left(X^{\prime}\right)$ on $\mathbb{R}^{n}$, then $u$ is superharmonic and

$$
u\left(Y^{\prime}, y\right)<\liminf _{\substack{X \rightarrow\left(Y^{\prime}, y\right) \\ X \in A^{\prime} \times \mathbb{R}}} u(X),
$$

whence again $A^{\prime} \times \mathbb{R}$ is thin at $\left(Y^{\prime}, y\right)$.

Conversely, suppose that $A^{\prime} \times \mathbb{R}$ is thin at $\left(Y^{\prime}, y\right)$, let $\omega^{\prime}$ denote the open cube in $\mathbb{R}^{n-1}$ of side length $1 / 2$ centred at $Y^{\prime}$, and let $v$ denote the Green function for $\omega^{\prime} \times \mathbb{R}$ with pole at $\left(Y^{\prime}, y\right)$. We extend $v$ to all of $\mathbb{R}^{n}$ by assigning it the value 0 elsewhere. Thus $v$ is subharmonic on $\mathbb{R}^{n} \backslash\left\{\left(Y^{\prime}, y\right)\right\}$. Noting that the function

$$
\cos \left(\pi x_{1}\right) \cos \left(\pi x_{2}\right) \cdots \cos \left(\pi x_{n-1}\right) \exp (-\sqrt{n-1} \pi|x|)
$$


is positive and superharmonic on $(-1 / 2,1 / 2)^{n-1} \times \mathbb{R}$, we see easily that

$$
v\left(X^{\prime}, x\right) \leq c \exp (-\sqrt{n-1} \pi|x-y|) \quad\left(X^{\prime} \in \omega^{\prime} ;|x-y|>1\right)
$$

for some positive constant $c$. In particular, the function $v^{\prime}$ defined by

$$
v^{\prime}\left(X^{\prime}\right)=\int_{-\infty}^{+\infty} v\left(X^{\prime}, x\right) d x \quad\left(X^{\prime} \in \mathbb{R}^{n-1}\right)
$$

is finite on $\mathbb{R}^{n-1} \backslash\left\{Y^{\prime}\right\}$. Further, it follows from [9, Theorems 1, 4] that $v^{\prime}$ is positive and superharmonic on $\omega^{\prime}$ and subharmonic on $\mathbb{R}^{n-1} \backslash\left\{Y^{\prime}\right\}$. Since $v^{\prime}=0$ on $\mathbb{R}^{n-1} \backslash \omega^{\prime}$, we conclude from Bôcher's theorem that $v^{\prime}=a s^{\prime}$ for some $a>0$, where $s^{\prime}$ is the Green function for $\omega^{\prime}$ with pole at $Y^{\prime}$. Let $w$ be the regularized reduced function (balayage) of $v$ relative to $\left(A^{\prime} \cap \omega^{\prime}\right) \times \mathbb{R}$ in $\omega^{\prime} \times \mathbb{R}$, and let $w^{\prime}\left(X^{\prime}\right)$ denote the integral of $w$ over $\left\{X^{\prime}\right\} \times \mathbb{R}$, for each $X^{\prime}$ in $\omega^{\prime}$. Then $w=v$ on $\left(A^{\prime} \cap \omega^{\prime}\right) \times \mathbb{R}$, except perhaps for the polar subset of $\left(A^{\prime} \cap \omega^{\prime}\right) \times \mathbb{R}$ where that set is thin. By translation invariance, this polar set is of the form $F^{\prime} \times \mathbb{R}$, and it follows from Lemma 1 that $F^{\prime}$ is polar in $\mathbb{R}^{n-1}$. Hence $w^{\prime}$ is a non-negative superharmonic function on $\omega^{\prime}$ which satisfies $w^{\prime} \leq v^{\prime}$ on $\omega^{\prime}$ and $w^{\prime}=v^{\prime}$ on $\left(A^{\prime} \cap \omega^{\prime}\right) \backslash F^{\prime}$. It follows that $w^{\prime} \geq a t^{\prime}$, where $t^{\prime}$ denotes the regularized reduced function of $s^{\prime}$ relative to $A^{\prime} \cap \omega^{\prime}$ in $\omega^{\prime}$. However, $w \neq v$ since $A^{\prime} \times \mathbb{R}$ is thin at $\left(Y^{\prime}, y\right)$. Thus the superharmonic functions $w$ and $v$ must differ on a set of positive $\lambda_{n}$-measure in $\omega^{\prime} \times \mathbb{R}$, and so $w^{\prime} \neq v^{\prime}$. Hence $t^{\prime} \neq s^{\prime}$, and it follows that $A^{\prime}$ is thin at $Y^{\prime}$. This completes the proof of Lemma 2.

We note that one implication in Lemma 2 can be reformulated as follows.

Proposition 1. The canonical projection from $\mathbb{R}^{n}$ to $\mathbb{R}^{n-1}$ is a fine open mapping.

To see this, let $W$ be a fine open subset of $\mathbb{R}^{n}$, let $W^{\prime}$ denote its projection onto $\mathbb{R}^{n-1}$ and let $X^{\prime} \in W^{\prime}$. Then there exists $x$ in $\mathbb{R}$ such that $\left(X^{\prime}, x\right) \in W$. The set $\left(\mathbb{R}^{n-1} \backslash W^{\prime}\right) \times \mathbb{R}$, being a subset of $\mathbb{R}^{n} \backslash W$, is thin at $\left(X^{\prime}, x\right)$. Hence, by Lemma 2 , $\mathbb{R}^{n-1} \backslash W^{\prime}$ is thin at $X^{\prime}$. It follows that $W^{\prime}$ is a fine open subset of $\mathbb{R}^{n-1}$.

2.2. We will now prove Theorem 3 using Lemma 2 and an argument of Hayman $[13$, pp. 472, 473]. Let $g: D \rightarrow[-\infty,+\infty]$ be fine continuous, let

$$
E^{\prime}=\left\{X^{\prime} \in \mathbb{R}^{n-1}: C_{F}\left(g, X^{\prime}\right) \backslash C_{N}\left(g, X^{\prime}\right) \neq \varnothing\right\},
$$

and let $\mathcal{I}$ denote the family of closed intervals of $[-\infty,+\infty]$ with endpoints in $\mathbb{Q} \cup\{-\infty,+\infty\}$. Now let $Y^{\prime} \in E^{\prime}$. Noting that $C_{N}\left(g, Y^{\prime}\right)$ is a compact subset of $[-\infty,+\infty]$, we can find $I$ in $\mathcal{I}$, a finite union $J$ of intervals from $\mathcal{I}$, and a positive rational number $q$ such that $I \cap C_{F}\left(g, Y^{\prime}\right) \neq \varnothing$, such that

$$
\left\{g\left(Y^{\prime}, y\right): 0<y<q\right\} \subseteq J,
$$

and such that $I \cap J=\varnothing$. If $I, J$ and $q$ are as above, then we will say that $Y^{\prime} \in E^{\prime}(I, J, q)$. Thus

$$
E^{\prime} \subseteq \bigcup_{I, J, q} E^{\prime}(I, J, q)
$$

where the union is over all possible choices of $I, J$ and $q$ as described above.

Now suppose that one of these sets, $E^{\prime}\left(I_{0}, J_{0}, q_{0}\right)=A^{\prime}$ say, has the property that its fine closure $F^{\prime}$ has non-empty fine interior $V^{\prime}$, and let $X^{\prime} \in F^{\prime}$. Then the fine closure of $A^{\prime} \times\left(0, q_{0}\right)$ contains $F^{\prime} \times\left(0, q_{0}\right)$, by Lemma 2 . Hence, by fine continuity, $g\left(X^{\prime}, x\right) \in J_{0}$ whenever $\left(X^{\prime}, x\right) \in F^{\prime} \times\left(0, q_{0}\right)$. The set $V^{\prime} \cap A^{\prime}$ is non-empty, so we 
can choose a point $Z^{\prime}$ in it. Then $V^{\prime} \times\left(-q_{0}, q_{0}\right)$ is a fine neighbourhood of $\left(Z^{\prime}, 0\right)$, by Lemma 2 , and so

$$
C_{F}\left(g, Z^{\prime}\right) \subseteq J_{0} \subseteq[-\infty,+\infty] \backslash I_{0} .
$$

This contradicts the fact that $I_{0} \cap C_{F}\left(g, Z^{\prime}\right) \neq \varnothing$. Thus each set $E^{\prime}(I, J, q)$ must have the property that its fine closure has empty fine interior. It follows that $E^{\prime}$ is of first fine category, and so Theorem 3 is proved.

2.3. Corollary 2 follows from Theorem 3 and the fact (see $[11, \S 6]$ ) that, if a subset $A$ of $D$ is thin at a boundary point $\left(X^{\prime}, 0\right)$, then $A$ is minimally thin at $\left(X^{\prime}, 0\right)$ with respect to $D$.

\section{Proof of Theorem 1 and Corollary 1}

3.1. Let $u$ and $U^{\prime}$ be as in the statement of Theorem 1, let

$$
E^{\prime}=\left\{X^{\prime} \in U^{\prime}: \limsup _{x \rightarrow 0+} u\left(X^{\prime}, x\right)<+\infty\right\},
$$

and let

$$
E_{j, k}^{\prime}=\left\{X^{\prime} \in U^{\prime}: u\left(X^{\prime}, x\right) \leq j \text { when } 0<x<k^{-1}\right\} \quad(j, k \in \mathbb{N}) .
$$

Then

$$
E^{\prime}=\bigcup_{j, k} E_{j, k}^{\prime}
$$

Suppose that $E^{\prime}$ is not of first fine category. Then there exist $j_{0}$ and $k_{0}$ such that the fine closure of $E_{j_{0}, k_{0}}^{\prime}$ has non-empty fine interior $V^{\prime}$. Since $u$ is fine continuous, we can use Lemma 2 (as we did in $\S 2.2$ ) to see that $u \leq j_{0}$ on $V^{\prime} \times\left(0, k_{0}^{-1}\right)$. Hence (cf. §2.3) the open set $\left\{X \in D: u(X)<j_{0}+1\right\}$ is a deleted minimal fine neighbourhood of each point of $V^{\prime} \times\{0\}$. A result of Doob [6, Theorem 5.1] now shows that $u$ has finite minimal fine limits at $\lambda_{n-1}$-almost every point of $V^{\prime} \times\{0\}$. Since $C_{N}\left(u, X^{\prime}\right) \subseteq C_{M}\left(u, X^{\prime}\right)$ for $\lambda_{n-1}$-almost every $X^{\prime}$ in $\mathbb{R}^{n-1}$ (see $\S 1$ ), we deduce that

$$
\lambda_{n-1}\left(\left\{X^{\prime} \in V^{\prime}: \liminf _{x \rightarrow 0+} u\left(X^{\prime}, x\right)=-\infty\right\}\right)=0 .
$$

Hence, since $V^{\prime} \cap U^{\prime}$ is non-empty and fine open, the set

$$
\left\{X^{\prime} \in \mathbb{R}^{n-1}: \liminf _{x \rightarrow 0+} u\left(X^{\prime}, x\right)=-\infty\right\}
$$

is not metrically fine dense in $U^{\prime}$. This completes the proof of Theorem 1 .

3.2. Corollary 1 follows from Theorem 1 in view of the following two facts.

(I) Any fine open set $U$ in $\mathbb{R}^{n}$ has positive $\lambda_{n}$-measure. To see this, let $B$ be an open ball centred at a point $X$ of $U$, let $v$ be the Green function for $B$ with pole at $X$, and let $w$ be the regularized reduced function of $v$ relative to $B \backslash U$ in $B$. Then $w \not \equiv v$, so $w \neq v$ on a set of positive $\lambda_{n}$-measure, but $w=v \lambda_{n}$-almost everywhere on $B \backslash U$.

(II) The fine topology on $\mathbb{R}^{n}$ has the Baire property; that is, the intersection of a countable collection of fine open fine dense sets is fine dense (see [8, 1.XI.1]). 


\section{Proof of Theorem 2}

Let $f, u$ and $E^{\prime}$ be as in the statement of Theorem 2. There is no loss of generality in assuming that $f$ is continuous (see $[13, \S 4]$ ). The function $g$ defined by $g\left(X^{\prime}, x\right)=$ $u\left(X^{\prime}, x\right)-f(x)$ is then fine continuous on $D$. Let

$$
E_{j, k}^{\prime}=\left\{X^{\prime} \in \mathbb{R}^{n-1}: u\left(X^{\prime}, x\right) \leq j+f(x) \text { when } 0<x<k^{-1}\right\} \quad(j, k \in \mathbb{N}) .
$$

Thus

$$
E^{\prime}=\bigcup_{j, k} E_{j, k}^{\prime} .
$$

As in $\S 3.1$ we observe that, if $E^{\prime}$ is not of first fine category, then there exist $j_{0}, k_{0}$ and a non-empty fine open subset $V^{\prime}$ of $\mathbb{R}^{n-1}$ such that

$$
u\left(X^{\prime}, x\right) \leq j_{0}+f(x) \quad\left(X^{\prime} \in V^{\prime} ; 0<x<k_{0}^{-1}\right) .
$$

This contradicts Corollary 1, so Theorem 2 is established.

\section{An example}

The following example (cf. [13, Theorem 6]) illustrates Theorems 1 and 2 .

Example. There is a subset $A^{\prime}$ of $\mathbb{R}^{n-1}$ such that:

(i) $A^{\prime}$ is of first fine category and $\lambda_{n-1}\left(\mathbb{R}^{n-1} \backslash A^{\prime}\right)=0$;

(ii) for any continuous function $f:(0,1] \rightarrow \mathbb{R}$ there is a harmonic function $u$ on $D$ such that

$$
\limsup _{x \rightarrow 0+}\left\{u\left(X^{\prime}, x\right)-f(x)\right\} \leq 0 \quad\left(X^{\prime} \in A^{\prime}\right)
$$

(iii) there is a negative subharmonic function $v$ on $D$ which has limit $-\infty$ at each point of $\left(\mathbb{R}^{n-1} \backslash A^{\prime}\right) \times\{0\}$.

To see this, let $\left(G_{k}\right)$ be a decreasing sequence of dense open subsets of $\mathbb{R}$ such that $\lambda_{1}\left(G_{k}\right)<k^{-1}$, let $A_{k}^{\prime}=\left(\mathbb{R} \backslash G_{k}\right) \times \mathbb{R}^{n-2}$ and $A^{\prime}=\bigcup_{k} A_{k}^{\prime}$. Clearly $\lambda_{n-1}\left(\mathbb{R}^{n-1} \backslash A^{\prime}\right)=$ 0 . Also, $G_{k} \times \mathbb{R}^{n-2}$ is open and non-thin at each point of $\mathbb{R}^{n-1}$, so $A^{\prime}$ is of first fine category. Thus (i) holds. The set

$$
E=\bigcup_{k=1}^{\infty}\left(A_{k}^{\prime} \times\left(0, k^{-1}\right]\right)
$$

is relatively closed in $D$, and it is easy to see that $D \backslash E$ is non-thin at each point of $E$. Further, if $D^{*}$ denotes the Alexandroff (one-point) compactification of $D$, then $D^{*} \backslash E$ is connected and locally connected. It now follows from a result of Shaginyan [14] (or see [10, Theorem 3.19]) that, given any continuous function $f:(0,1] \rightarrow \mathbb{R}$ there is a harmonic function $u$ on $D$ such that

$$
\left|u\left(X^{\prime}, x\right)-(f(x)-1)\right|<1 \quad\left(\left(X^{\prime}, x\right) \in E\right) .
$$

Hence (ii) holds. Finally, (iii) follows from the fact that $\left(\mathbb{R}^{n-1} \backslash A^{\prime}\right) \times\{0\}$ has zero harmonic measure for $D$ (see $[8,1$.VIII.5(b)]). 


\section{REFERENCES}

1. M. G. Arsove, The Lusin-Privalov theorem for subharmonic functions, Proc. London Math. Soc. (3) 14 (1964), 260-270. MR 28:4136

2. K. F. Barth and W. J. Schneider, An asymptotic analogue of the F. and M. Riesz radial uniqueness theorem, Proc. Amer. Math. Soc. 22 (1969), 53-54. MR 40:364

3. R. D. Berman, Analogues of radial uniqueness theorems for subharmonic functions in the unit disk, J. London Math. Soc. (2) 29 (1984), 103-112. MR 85d:31003

4. E. F. Collingwood and A. J. Lohwater, The theory of cluster sets, Cambridge University Press, 1966. MR 38:325

5. J. Deny and P. Lelong, Étude des fonctions sousharmoniques dans un cylindre ou dans un cône, Bull. Soc. Math. France 75 (1947), 89-112. MR 9:352e

6. J. L. Doob, A non-probabilistic proof of the relative Fatou theorem, Ann. Inst. Fourier (Grenoble) 9 (1959), 293-300. MR 22:8233

7. J. L. Doob, Some classical function theory theorems and their modern versions, Ann. Inst. Fourier (Grenoble) 15 (1965), 113-136; 17 (1967), 469. MR 34:2923; MR 36:4013

8. J. L. Doob, Classical potential theory and its probabilistic counterpart, Springer, New York, 1983. MR 85k:31001

9. S. J. Gardiner, Integrals of subharmonic functions over affine sets, Bull. London Math. Soc. 19 (1987), 343-349. MR 88g:31005

10. S. J. Gardiner, Harmonic approximation, London Math. Soc. Lecture Note Ser., no. 221, Cambridge University Press, 1995. CMP 95:15

11. J. Lelong-Ferrand, Étude au voisinage de la frontière des fonctions surharmoniques positives dans un demi-espace, Ann. Sci. École Norm. Sup. (3) 66 (1949), 125-159. MR 11:176f

12. N. Lusin and I. Privalov, Sur l'unicité et la multiplicité des fonctions analytiques, Ann. Sci. École Norm. Sup (3) 42 (1925), 143-191.

13. P. J. Rippon, The boundary cluster sets of subharmonic functions, J. London Math. Soc. (2) 17 (1978), 469-479. MR 81h:30036

14. A. A. Shaginyan, Uniform and tangential harmonic approximation of continuous functions on arbitrary sets, Mat. Zametki 9 (1971), 131-142; English translation in Math. Notes 9 (1971), 78-84. MR 45:2375

Department of Mathematics, University College, Dublin 4, Ireland

E-mail address: gardiner@acadamh.ucd.ie 